\title{
Small fiber neuropathy in unexpected clinical settings: a review.
}

\author{
Majid Ghasemi ${ }^{1,2}$ MD \\ Yusuf A. Rajabally ${ }^{1,3}$ MD FRCP \\ 1. Regional Neuromuscular Service, University Hospitals Birmingham, Birmingham, U.K. \\ 2. Department of Neurology, School of Medicine, Isfahan University of Medical Sciences, Isfahan, \\ Iran. \\ 3. Aston Medical School, Aston University, Birmingham, U.K.
}

\section{INVITED REVIEW REVISED VERSION R3}

\section{Running Head: Unexpected small fiber neuropathy}

Key words: amyotrophic lateral sclerosis; inflammatory neuropathy; obstructive sleep apnea syndrome; Parkinson disease; REM sleep behavior disorder; small fiber neuropathy; vaccination.

\section{Disclosures:}

MG has no disclosures.

YAR has received speaker/consultancy honoraria from CSL Behring, LFB, Grifols, BPL, Octapharma and Kedrion, has received educational sponsorships from LFB, CSL Behring and Baxter and has obtained research grants from CSL Behring and LFB.

Ethical Publication Statement: We confirm that we have read the Journal's position on issues involved in ethical publication and affirm that this report is consistent with those guidelines.

\section{Funding: None}

Correspondence to:

Yusuf A. Rajabally

Aston Medical School,

This article has been accepted for publication and undergone full peer review but has not been through the copyediting, typesetting, pagination and proofreading process which may lead to differences between this version and the Version of Record. Please cite this article as doi: $10.1002 /$ mus. 26808 
Aston University,

Aston Triangle,

Birmingham B4 7ET, UK.

E-mail: y.rajabally@aston.ac.uk

\section{Abstract.}

Small fiber neuropathy (SFN) is being recognized with increasing frequency in neuromuscular practice due to improved diagnostic techniques. Although there are some common etiologies, up to one-third of cases are considered idiopathic. In recent years, several disorders have unexpectedly been reported in association with SFN, on clinical grounds and complementary investigations, including quantitative sensory testing, intraepidermal nerve fiber density and confocal corneal microscopy. Knowledge of these disorders is important in clinical practice as increased awareness enables prompt diagnosis of SFN in these settings and early optimal therapeutic management of affected patients. Furthermore, these new developments may lead to a better understanding of the pathophysiologic mechanisms underlying SFN in these different disorders as well as, in some cases, an expanded spectrum of affected organs and systems. This article reviews these reported associations, their possible pathophysiologic bases, and the potential resulting management implications. 


\section{Introduction.}

Small fiber neuropathy (SFN) is a sensory peripheral nerve disorder with structural and functional abnormalities involving the small myelinated $(\mathrm{A} \delta)$ and unmyelinated $(\mathrm{C})$ fibers and characterized pathologically by their degeneration (1). A $\delta$ fibers transmit temperature and pain sensation after mechanical or thermal stimulation, but $\mathrm{C}$ fibers in addition to pain and temperature, are also involved in autonomic function $(2,3)$. SFN may present with or without autonomic dysfunction in association with pain and sensory loss. The incidence and prevalence of SFN is not well established. Due to lack of awareness together with a paucity of readily available diagnostic methods, SFN is probably under-recognized. In a crosssectional Dutch study, the overall minimum incidence has been reported as approaching $12 / 100,000$ per year and the overall minimum prevalence, about 53/100,000 (4). In terms of morbidity and disease burden, peripheral neuropathy is reported to be the leading cause of outpatient neurology appointments in the United States and accounts for more than \$10 billion in healthcare cost each year (5). Also, chronic neuropathic pain is reported in almost $7 \%$ of adults (6). 
The precise pathophysiologic mechanisms underlying SFN are often unknown and depend on the underlying etiology, including oxidative stress, ischemia, direct neurotoxic effect, hypoxia, and inflammatory mediators (7-9). The cause of SFN is not always identifiable. It may be as a result of diverse disorders, including metabolic, inflammatory, toxic, infectious, and genetic diseases. Diabetes (10) and impaired glucose tolerance are probably the most common $(8,9)$. Impaired glucose tolerance may contribute to $15 \%$ of cases $(11)$. Other possible reported etiologies include connective tissue disease, HIV and hepatitis C infections, thyroid dysfunction, vitamin B12 deficiency, multiple myeloma and monoclonal gammopathy, amyloidosis, alcohol or drugs, and genetic. Recently, new hereditary forms of SFN have been described with pathogenic mutations in sodium channels [Nav1.7, Nav1.8, and Nav1.9] which lead to hyperexcitability of dorsal root ganglia and sensory axons. These gain-of-function mutations result in degeneration of small fibers (12). In a recent study on 921 patients with pure SFN, over 50\% of cases were of undetermined cause (13).

The diagnosis of SFN is now possible via different modalities. Quantitative sensory testing (QST) is a psychophysical investigation, using automated systems to deliver sensory stimuli in a quantifiable and reproducible manner. QST is highly patient-dependent and cannot be used to distinguish central from peripheral nervous system lesions (14). Skin biopsy with intraepidermal nerve C fiber density (IENFD) evaluation was developed and has been utilized for identification of SFN since the 1990s, greatly facilitating diagnosis. Counting rules have been established (15) and normative values derived, making IENFD measurement highly 
reproducible (16). Confocal corneal microscopy (CCM) is a novel, non-invasive method to detect SFN, exploring the cornea, which receives the densest small fiber innervations of the body. The method correlates well with IENFD and has in recent years been found to be helpful in identifying SFN in a number of different clinical settings (17).

Awareness of causes of SFN is important, as some are potentially treatable or curable. Conversely, several conditions have unexpectedly been reported recently to be accompanied by SFN. These conditions include a heterogeneous group of neurological and nonneurological disorders in which small fiber involvement is unpredictable. A list of these disorders, both neurological and non-neurological, is provided in Table 1.

Knowledge of these associations has become important for prompt recognition, as well as for early, adequate multidisciplinary management while in addition, potentially offering new insights in the pathophysiology of SFN. The aim of this review is to describe these newlyreported associations of unexpected reported conditions associated with SFN. 


\section{Methods.}

A search was performed using the PubMed database between January 1990 to December 2019, for “small fiber/fibre neuropathy/pathology”. We included "small fiber/fibre pathology” (SFP) as the concept used to designate findings of small fiber damage in association with diseases not characterized by the clinical features of small fiber neuropathy, identified in disorders of different pathophysiology (18), as well as the presence of SFP as the 
cause of painful disorders not previously known to have a neuropathic basis (19). Only English language publications were selected, and Abstracts were excluded. All case reports and original articles that were related to the combination of SFN and other neurologic/nonneurologic conditions, except for commonly reported causes, were extracted and evaluated. Publications that were solely expert editorials or commentaries were excluded. Reports related to well-known causes of neuropathies such as glucose metabolism disorders, connective tissue disorders, ion channel mutations, were excluded.

\section{Results.}

\section{Fibromyalqia}


Fibromyalgia (FM) (20) is a chronic widespread pain disorder with additional, non-specific symptoms such as fatigue, headache, cognitive dysfunction, mood disorder and sleep disturbances. This syndrome not only has a major effect on quality of life but also carries a substantial socio-economic burden. Diagnosis is based on the existence of widespread pain for at least three months in absence of a disorder that would otherwise explain the pain (21). Diagnostic criteria have been published (21), and used for inclusion by the studies described below.

The pathophysiologic basis of FM remains a matter of debate (22). There are considerable clinical and paraclinical data that suggest central nervous system (CNS) alterations in patients with FM (23). More recently, peripheral nervous system (PNS) abnormalities have also been described. Inflammatory changes on skin biopsies were initially reported (24), followed by IENFD reduction in a proportion of FM patients (25). A PNS role in the pathophysiology of FM was suggested in a subgroup of FM patients with a chronic polyneuritis similar to chronic inflammatory demyelinating polyneuropathy (CIDP) partially responding to immunoglobulin therapy (26). Reduced intra-epidermal nerve fiber density (IENFD) on skin biopsy indicative of small fiber involvement was subsequently described in patients with FM compared to healthy controls. Table 2 summarizes studies of SFN in fibromyalgia. The first report on nerve fiber density in FM was published in 2013 (27). In this study patients with FM were investigated with pain-related evoked potentials, IENFD in skin punch biopsies and quantitative sensory testing (QST), and compared to healthy controls and patients with 
monopolar depression. Patients with FM had impaired small fiber function in QST and painrelated evoked potentials, as well as significant reductions in dermal unmyelinated nerve fibers in skin samples from the lower leg and upper thigh. Abnormality of all three methods was not seen in all patients, but every patient at least had one abnormal value. These findings pointed to a pathological process involving small nerve fibers in FM. In another study, the IENFD in pure FM, FM plus autoimmune disorders and healthy controls were studied (28). This analysis showed that almost $26 \%$ of pure FM patients and around $44 \%$ of FM patients with another autoimmune disorder have reduced IENFD, in comparison to controls. In a further case-control study of patients with pure FM, mean values for both calf and thigh specimens IENFD were significantly lower in patients with FM than in controls. In addition, they underwent pinwheel and vibratory testing for hypoesthesia and all had varying degrees of stocking distribution diminished perception in the lower extremities (29). An electron microscopy study provided further evidence of small fiber pathology in FM showing diminished nerve fiber diameter in skin biopsies of FM patients compared with controls (30). In another recent study, SFN was reported as identified in patients with FM through sensory nerve conduction studies and IENFD measurements but not through pain features and intensity (31). The relevance of clinical SFN symptoms was however suggested by findings of other authors who described dysautonomia as well as paresthesia as predictors of SFN in patients with FM (32). Corneal confocal microscopy (CCM) is another diagnostic tool used for SFN in FM (33). It is a novel non-invasive method to detect SFN, exploring the cornea, which receives the densest small fiber innervations in the body. Other studies have demonstrated alterations of small fiber morphology in patients with FM by CCM (34). Total 
nerve density, long nerve fibers, and the number of nerves have all been found to be lower in patients with FM than in controls (35). Recently, aberrant microRNA (miR-let-7d) has been reported in the skin of patients with FM and SFN, correlating with reduced small nerve fiber density (36). MicroRNAs have been shown to be involved in a wide range of biological processes such as neurogenesis. These findings reinforce the concept that pain in some but not all patients with FM has a neuropathic source and point to the role of peripheral components. A very recent controlled study of 117 women with FM found reduced IENFD at different biopsy sites in 63\% versus 10\% in women with major depression and chronic widespread pain and $18 \%$ in healthy controls ( $\mathrm{p}<0.001$ versus both) (37). Compared to FM patients with normal skin innervation, those with IENFD reduction had higher pain intensity $(\mathrm{p}<0.05)$, providing new evidence of a relation between pain severity and SFP in FM.

Diagnostically, the latest meta-analysis of reported studies estimated the pooled prevalence of SFP at $49 \%$ in patients with FM. The prevalence estimate achieved by CCM was greater than that with skin biopsy (59\% vs. 45\%), suggesting the former may be a more sensitive method (38). The above findings, taken together, may suggest FM is a heterogenous condition with a complex pathobiology and that patients reside along a continuum with at one end a purely peripherally-induced and the other end a mainly purely centrally-induced form, and some patients in between (39). Of note, non-length-dependent SFN may underlie some presentations of FM (40). 


\section{Parkinson disease.}

Parkinson disease (41) is a progressive neurodegenerative disorder characterized by

predominant motor signs in conjunction with now well-recognized non-motor features (42). Neuropathy was noted in PD from reports on large fiber involvement associated with intrajejunal levodopa therapy. Subsequently, different studies demonstrated the higher prevalence of peripheral neuropathy in PD patients, indicating both large and small fiber involvement. The prevalence of large fiber neuropathy in PD was variable between studies, possibly due to methodology and gold standards of diagnosis, but all showed significantly greater prevalence in PD patients compared to controls. Several etiologies have been considered for neuropathy in PD including antiparkinsonian drugs (L-dopa), inducing or occurring concurrently with vitamin B12 or folate deficiency $(43,44)$. PNS involvement may otherwise constitute an intrinsic part of the neurodegenerative pathology of PD.

In favor of this hypothesis, several studies have demonstrated the early presence of alphasynuclein deposition in small nerve fibers in skin samples. Table 3 summarizes the published literature on the association of SFN with PD. Another recent small study reported 4 patients with PD with restless legs syndrome (RLS) as a presenting symptom prior to emerging of motor symptoms. Skin biopsy showed reduction of IENFD in both proximal and distal sites indicative of non-length dependent SFN in all 4 patients (45). Pain and sensory neuropathic symptoms are common non-motor symptom in PD, which may imply small fiber 
involvement. In another recent case-control study, patients with idiopathic PD and age-and sex-matched controls were screened for pain by King’s Parkinson’s Pain Scale and Michigan Neuropathy Screening Instrument. The reported pain prevalence was similar in both groups, but peripheral neuropathic pain (allodynia, hyperalgesia, burning pain) was significantly more prevalent in PD patients than controls and correlated with duration of disease and years on levodopa treatment (46). Importantly, confirmation of a peripheral neuropathic origin of the pain manifestations in PD requires a correlation with objective markers of small fiber nerve damage. On the other hand, sensory impairment as well as loss of free and encapsulated nerve endings has been shown in patients with PD, evident in all examined sites, unrelated to age, disease duration or levodopa therapy (47). Another study demonstrated in $>95 \%$ of PD patients, at least one abnormality in IENF, contact heat evoked potentials, or thermal thresholds of the foot, suggesting small fiber dysfunction (48). In a further analysis, treatment-naïve PD patients were evaluated by electrophysiology, skin biopsy and CCM and compared to normal controls. This study showed no significant difference between groups in terms of nerve conduction studies and IENFD, but CCM showed significant reduction of corneal nerve fiber density and length in PD patients (49). Both skin biopsy and CCM was used in another study. Corneal nerve pathology was demonstrated in PD-affected subjects, which had a correlation with autonomic symptoms, motor function, and parasympathetic deficit. This study found that IENFD was also reduced and correlated with corneal fiber density and motor symptoms (50). Finally, one study showed by skin biopsy that in both naïve and L-dopa treated patients, small fiber impairment occurs in early stage of disease (51). An important finding in this analysis was the 
asymmetric loss of IENFD which was greater on the side more severely affected by the extrapyramidal syndrome, which may suggest that SFN forms part of the underlying disease process in PD. Also, alpha-synuclein deposition has been found to be increased in cutaneous sympathetic but not in sensory neurons suggesting this may not underlie the sensory and in particular painful symptoms (52).

The issue of pain remains complex in PD. The neuropathic nature of pain is uncertain in all cases and is highlighted by some studies (46). The presence of SFP in PD is illustrated by the studies above, although its association with pain of definite neuropathic origin in subjects with $\mathrm{PD}$, rather than any pain, will require further detailed clinical and histopathological studies.

\section{Other synucleionopathies.}

REM sleep behavior disorder (RBD) is a parasomnia characterized by loss of muscle tone during REM sleep accompanied by abnormal behaviors often occurring with dreams. RBD can be symptomatic of $\alpha$-synucleinopathies (Parkinson disease, Lewy body dementia, multiple system atrophy) (53). Recent data suggest that cutaneous changes could be a histopathological window and probably a diagnostic tool for neurodegenerative $\alpha$ synucleinopathies (54). Patients with RBD may therefore be expected to demonstrate the cutaneous abnormalities of SFN. In keeping with this, impairment of somatosensory function with increased thermal detection thresholds has been shown in patients with idiopathic RBD 
(55). In addition, skin biopsy of RBD patients vs. controls demonstrated a significant reduction of IENFD, suggesting early PNS involvement (56). Clinical correlation is yet uncertain, but recognition of SFN in this setting appears important in view of the growing evidence for this association.

\section{Amyotrophic lateral sclerosis (ALS)}

There is growing evidence for sensory system involvement in ALS (57). On the other hand, pain is recognized as a prevalent complaint in ALS with various mechanisms including neuropathic and can be associated with poorer functional status (58) and longer disease duration (59). It is important in this regard to recognize the important and wider and issue of pain in ALS, with its numerous origins and mechanisms, often unrelated to SFN (60).

Table 4 summarizes the published studies describing the presence of SFN in ALS. One early study on post-mortem tissue found nerve growth factor receptor immunostaining in mixed peripheral nerves and in dorsal roots, suggesting ALS was not a pure motor disorder (61). Sural nerve biopsies in 17 cases of sporadic ALS showed different degrees of axonal loss in about $70 \%$ of cases. However, these patients with ALS were clinically atypical due to the presence of sensory symptoms (62). This suggests that these patients may have had an associated sensory neuropathy of undetermined cause. A further case-control study demonstrated sural nerve abnormalities in 91\% of ALS patients without co-existing disease (63). Large-caliber myelinated fibers were predominantly affected in three-quarters of cases 
with small-caliber myelinated fibers affected in only about a quarter. Thinly myelinated fibers were present in $95 \%$ and regenerating clusters in $77 \%$ of the biopsies.

An initial study of skin biopsies of sporadic ALS patients showed reduction of IENFD in almost $80 \%$ of patients vs. $12 \%$ of controls as well as a reduction in subepidermal and sweat gland innervation (64). Another study using QST and skin biopsy demonstrated that peripheral sensory nerve involvement in ALS differs according to mode of onset. Patients with spinal-onset but not those with bulbar-onset ALS, had both abnormal thermal-pain perceptive thresholds and reductions of IENFD (65). However, this was not confirmed by a subsequent larger analysis which showed reduction of IENFD similarly in all ALS subtypes (66), with no less than 75\% of patients with pure ALS demonstrating reduced IENFD. In another recent study, in addition to reduced IENFD, Meissner's corpuscle density, sweat gland nerve fiber density as well as pilomotor nerve fiber density were all also reduced. Interestingly also, small fiber symptomatology was greater in ALS patients that were pTDP43 positive than in those who were pTDP-43 negative (67). This may raise the issue of genetic susceptibility for concurrent small fiber pathology in some forms of ALS, but also of PD. We are not aware of reported evidence of SFP in familial ALS. Small fiber involvement has also been observed by CCM in ALS. CCM showed that corneal nerve fiber damage significantly correlated with bulbar disability scores, but not with age, disease duration or spinal level disability (68).

\section{Ehlers Danlos syndrome}


Ehlers-Danlos syndrome (EDS) describes different inherited connective tissue disorders with joint hypermobility, skin texture abnormalities, and visceral and vascular fragility and dysfunction (69). A number of different subtypes are reported. An initial questionnaire-based analysis found a likely neuropathic component to pain as described in detail (70), with another suggesting the responsibility of compressive and axonal neuropathies in the hyperalgesia of EDS (71). One subsequent study revealed high prevalence of neuropathic pain and of decreased IENFD on skin biopsy, consistent with SFN, in patients with EDS (72). This has however, not been replicated in other cohorts, to our knowledge.

\section{Vaccination and SFN}

Human papillomavirus (HPV) vaccination is reported with more adverse reactions than other vaccines (73). Common neurologic complications after HPV vaccination have been complex regional pain syndrome, postural orthostatic tachycardia syndrome (POTS), and FM, all reported with concurrent small fiber neuropathy. In one report, three out of six cases of POTS after HPV vaccination had small fiber neuropathy diagnosed by QST (74). Another report also describes 5 cases of SFN confirmed by skin biopsy following vaccination for rabies, varicella zoster, or Lyme disease (75). These case reports suggested that acute or subacute SFN may occasionally follow immunization by other common vaccines.

\section{SFN and inflammatory neuropathies}


In inflammatory demyelinating polyneuropathies (Guillain-Barré syndrome [GBS]; chronic inflammatory demyelinating polyneuropathy [CIDP]), large fibers represent the main target of the immunologic attack (76). However, there is some evidence confirming the coexisting involvement of small fibers in these disorders (77). Especially in patients with GBS, sensory signs and symptoms such as hypoesthesia, neuropathic pain and allodynia, in addition to autonomic dysfunction, raise the possibility of frequent small fiber pathology. In one study, patients with GBS were assessed by quantifying cold and warm detection and pain thresholds and responses to suprathreshold painful thermal and mechanical stimuli (QST). Significant impairment of these parameters was shown in GBS patients with neuropathic pain compared to those with non-neuropathic pain and without pain. Interestingly, motor and sensory large fiber dysfunction were similar between groups (78). The same method showed similar results in another cohort of patients with GBS, in addition early small fiber nerve involvement in those with slow recovery (79). Skin biopsy demonstrated significantly reduced mean IENFD in GBS patients compared to controls in a further study (80) where IENFD was reduced in $55 \%$ of cases. In addition, patients with GBS had significantly elevated thermal thresholds compared to controls. Reduced IENFD was associated with respiratory distress and dysautonomia, and correlated with disability. In addition, case series of acute-onset pure small fiber neuropathy as a variant of GBS have also been described. Six patients with small fiber neuropathy preceded by infection and associated with CSF albumin-cytologic dissociation were reported, all with favorable outcomes (81). Acute presentation of SFN may therefore represent part of the GBS spectrum. 
Similarly, small fiber involvement in CIDP has been shown in a few studies by QST and skin biopsy. An association was found between autonomic symptoms and IENFD reduction (82). Small fiber and autonomic functions have also been evaluated by quantitative methods. Autonomic symptoms were uncommon, but autonomic deficits as measured by the Composite Autonomic Severity Score (CASS) were frequent (47\%), though very mild, cholinergic and predominant sudomotor. QST showed small fiber abnormalities in $72 \%$ of patients. There was no association between the presence of small fiber abnormalities (QST) and definite autonomic deficits on CASS (83). There are few reports of painful small fiber neuropathy in association with anti-MAG antibody (84), although this may be underrecognized despite the frequent present of pain this disorder (85). Potassium/sodium hyperpolarization-activated cyclic nucleotide-gated ion channel 2, which is encoded by the HCN2 gene, has been identified as an important regulator of nociceptive pain (86). Voltagegated potassium channels (VGKC) work synergistically with these channels to maintain nociceptive afferent sensory neural thresholds. One of the recently discovered mechanisms of chronic idiopathic pain is small fiber nerve hyperexicitability, by involvement of these channels. Mutations of sodium channels (Nav1.7 \& Nav1.8) have been described in affected individuals of chronic pain with or without small fiber loss (87), but this nerve hyperexicitability may also result from autoimmunity against VGKC. Anti-VGKC antibodies were first described in Isaac’s and Morvan’s syndromes. Contactin Associated Protein 2 (CASPR2) is a subunit of the VGKC complex and is included in the group of extracellular antigens. In a large study of 54,853 patients with diverse neurologic presentations, pain was significantly associated with CASPR2-IgG-positivity, the antibody being detected in $16 \%$ of 
patients with pain and in only $7 \%$ without pain $(p=0.014)$. Pain was of subacute onset, but with a chronic course, both neuropathic and nociceptive, regional, or diffuse. Some patients had been diagnosed with FM (6\%) or as having psychogenic pain(13\%). Electrophysiologic studies in affected individuals were normal, suggestive of small fiber dysfunction, but skin biopsy was not performed (88). Finally, anti-GQ1b antibodies were recently described in a case of SFN in a 45-year-old woman. (89) 


\section{Discussion.}

A number of different conditions, not primarily considered as having an accompanying neuropathic component, have recently been described with SFN.

In the evaluation of neuropathy in neurologic practice, SFN is frequently suspected following clinical evaluation and a normal electrophysiological examination. The modalities of further targeted testing in this case scenario are now better established, with the increasing availability of skin biopsy in addition to QST, and with the advent of CCM. The findings of this review highlight the importance of considering the wider clinical setting, in addition to traditionally well-known etiologies of SFN such as diabetes, IGT or vitamin deficiencies. Although the frequencies of the different associations currently remain unknown, thorough clinical assessment through history-taking and examination, appears warranted given the possible associations or underlying extra-pyramidal, anterior horn cell or rheumatologic disease, all of which may equally require specialist input. Similarly, appropriate attention is 
required in presentations of neuropathic pain in subjects with known FM, PD, ALS, RBD or EDS. The literature reviewed suggests that it is important to keep in mind that SFN may be a part of a more complex disorder, rather than necessarily being considered a separate entity with only a limited number of causes. As discussed above with regard to PD, the issue of pain is frequently complex and requires careful consideration and separation of neuropathic from non-neuropathic origins.

In practice, the reported associations also have a number of implications. Focusing diagnostic and therapeutic management efforts solely on small fiber symptoms may be insufficient and directing the clinical assessment in search of features of possible concurrent disorders warrants increased emphasis. This may benefit from extended history-taking and more exhaustive examination with adequate consideration of potentially neglected nonneurological elements, such as joint pain or hypermobility. Similarly, given higher prevalence rates of the other possible concurrent disorders, therefore making them more likely to be encountered in general practice, greater attention to symptoms associated with SFN is warranted in patients with established other diagnoses. In such patients, appropriate additional investigations as well as consideration of treatment may be essential despite the known, main diagnosis. The examples of PD and ALS are particularly striking, as in both disorders, additional neuropathic pain represents an obvious cause of further quality of life impairment, which may be amenable to treatment. 
In conclusion, SFN is now recognized as being a more complex disorder, occurring in previously unrecognized settings, associated with other unexpected disorders, but of relatively straightforward diagnosis if a high index of suspicion is maintained. SFN is, most importantly, potentially treatable. Greater awareness of this growing literature appears essential for all clinical neurologists.

Table 1. Reported unexpected diseases associated with small fiber neuropathy.

\begin{tabular}{|l|l|}
\hline Neurological & Non-neurological \\
\hline Amyotrophic lateral sclerosis & Fibromyalgia \\
\hline Parkinson's disease & Ehlers Danlos Syndrome \\
\hline Rapid eye movement sleep behavior disorders & Obstructive Sleep Apnea Syndrome \\
\hline Pure Autonomic Failure & Vaccination \\
\hline Dementia with Lewy Bodies & Fragile X Syndrome \\
\hline
\end{tabular}




\begin{tabular}{|l|l|}
\hline Inflammatory Large Fiber Neuropathies & Leprosy \\
\hline Acute Intermittent Porphyria & Chagas Disease \\
\hline Pompe's Disease & Wilson's Disease \\
\hline
\end{tabular}

Table 2. Studies reporting SFN in Fibromyalgia.

\begin{tabular}{|l|l|l|l|}
\hline Study & case/control & Method & Results \\
\hline Doppler K.(30) & $32 / 24$ & Skin biopsy & $\downarrow$ mean axon diameter \\
\hline Giannoccaro MP.(25) & $20 / 0$ & Skin biopsy & $\downarrow$ IENFD ${ }^{1}$ (6 case) \\
\hline
\end{tabular}




\begin{tabular}{|c|c|c|c|}
\hline Erkan Turan K. (35) & $34 / 42$ & $\mathrm{CCM}^{2}$ & $\begin{array}{l}\uparrow \text { Ocular surface alterations } \\
\downarrow \text { Nerve density }\end{array}$ \\
\hline Manuel Ramírez (34) & $17 / 17$ & CCM & $\begin{array}{l}\downarrow \text { Mean stromal nerve thickness } \\
\downarrow \text { Mean sub-basal plexus nerve density }\end{array}$ \\
\hline Michalis L. Kosmidis(28) & $46 / 34$ & Skin biopsy & $\downarrow$ IENFD in $15 / 46$ \\
\hline Xavier J. Caro(29) & $41 / 47$ & Skin biopsy & $\downarrow$ Mean IENFD \\
\hline Üçeyler N.(27) & $25 / 10$ & $\begin{array}{l}\text { 1.Skin biopsy } \\
\text { 2.QST } \\
\text { 3.pain-related evoked } \\
\text { potentials }\end{array}$ & $\begin{array}{l}\text { 1. } \downarrow \text { Mean IENFD } \\
\text { 2.impaired small fiber function } \\
\text { 3. } \uparrow \text { N1 latencies upon stimulation and } \\
\downarrow \text { amplitudes of pain-related evoked potentials }\end{array}$ \\
\hline Oudejans L.(33) & $39 / 0$ & CCM & 51\% small fiber pathology \\
\hline Oaklander AL.(40) & $27 / 30$ & $\begin{array}{l}\text { 1.Skin biopsy } \\
\text { 2. } \text { MNSI }^{4} \\
\text { 3. } \text { UENS }^{5}\end{array}$ & $\begin{array}{l}\text { 1. } \downarrow \text { IENFD in } 41 \% \text { vs. } 3 \% \\
\text { 2. } \uparrow \text { Mean MNSI } \\
\text { 3. } \uparrow \text { Mean UENS }\end{array}$ \\
\hline
\end{tabular}

IENFD: Intraepidermal nerve fiber density, CCM: corneal confocal microscopy, QST: Quantitative sensory testing, MNSI: Michigan Neuropathy Screening Instrument, UENS: Utah Early Neuropathy Scale

\section{Table 3. Studies reporting SFN in Parkinson Disease.}




\begin{tabular}{|c|c|c|c|}
\hline Study & Case/control & Method & Results \\
\hline Podgorny PJ.(49) & $26 / 22$ & $\begin{array}{l}\text { 1.Skin biopsy } \\
\text { 2.UENS } \\
\text { 3.CCM }\end{array}$ & $\begin{array}{l}\text { 1. No difference in IENFD } \\
\text { 2.Abnormal UENS in } \\
38 \% / 23 \% \\
\text { 3. } \downarrow \text { Mean CNFL }\end{array}$ \\
\hline Giannoccaro MP. (90) & $22 / 11$ & $\begin{array}{l}\text { 1.(123) I-MIBG myocardial } \\
\text { scintigraphy } \\
\text { 2. Skin biopsy }\end{array}$ & Abnormal both in $91 \%$ \\
\hline Nolano M.(47) & $18 / 30$ & $\begin{array}{l}\text { 1. Skin biopsy } \\
\text { 2.QST }\end{array}$ & $\begin{array}{l}\text { 1. } \downarrow \text { Mean IENFD } \\
2 . \uparrow \text { tactile and } \\
\text { thermal threshold } \\
\downarrow \text { mechanical pain } \\
\text { perception }\end{array}$ \\
\hline Nolano M. (51) & 85 & $\begin{array}{l}\text { 1. Skin biopsy } \\
\text { 2.QST }\end{array}$ & $\begin{array}{l}\text { 1. } \downarrow \text { Mean IENFD, higher in } \\
\text { the more affected side } \\
\text { 2. } \uparrow \text { tactile and thermal } \\
\text { thresholds, impairment of } \\
\text { mechanical pain perception, } \\
\text { and } \downarrow \text { sweat output }\end{array}$ \\
\hline Kass-Iliyya L. (50) & $26 / 26$ & $\begin{array}{l}\text { 1. Skin biopsy } \\
\text { 2.CCM }\end{array}$ & $\begin{array}{l}\text { 1. } \downarrow \text { IENFD } \\
\text { 2. } \downarrow \text { CNFD, } \uparrow \mathrm{CNBD}, \\
\uparrow \mathrm{CNFL}\end{array}$ \\
\hline Dabby R. (91) & $22 / 19$ & Skin biopsy & $\begin{array}{l}\downarrow \text { blood vessels, sweat } \\
\text { glands, and erector pili } \\
\text { muscles autonomic } \\
\text { innervation }\end{array}$ \\
\hline Nolano M. (92) & 28 & $\begin{array}{l}\text { 1.SFN-SIQ and SCOPA- } \\
\text { AUT } \\
\text { 2.neurographic study }\end{array}$ & $\begin{array}{l}\text {-changes in sensory and } \\
\text { autonomic function }\end{array}$ \\
\hline
\end{tabular}


CNFL: Corneal nerve fiber length, CNFD: Corneal nerve fiber density, CNBD: corneal nerve branch density, SFN-SIQ: small-fiber neuropathy- Symptoms Inventory Questionnaire, SCOPA-AUT: Scales for Outcomes in Parkinson disease- Autonomic questionnaire 
Table 4. Studies reporting SFN in ALS

\begin{tabular}{|c|c|c|c|}
\hline Study & Case/control & Method & Results \\
\hline Truini A.(65) & $24 / 0$ & $\begin{array}{l}\text { 1.QST } \\
\text { 2. Skin biopsy }\end{array}$ & $\begin{array}{l}\text { 1. Both normal in } \\
\text { bulbar-onset } \\
\text { 2. Both abnormal in } \\
\text { spinal-onset }\end{array}$ \\
\hline Ren Y.(67) & $18 / 18$ & Skin biopsy & $\begin{array}{l}\downarrow \text { Mean IENFD, MCD, } \\
\text { SGNFD, PNFD }\end{array}$ \\
\hline Hammad M.(63) & 103 & $\begin{array}{l}\text { 1. Nerve biopsy ( } 22 \\
\text { case/12 control) } \\
\text { 2. Electrodiagnostic } \\
\text { studies } \\
\text { 3. Clinical evaluation }\end{array}$ & $\begin{array}{l}\text { 1. } \downarrow \text { Large fiber in } 73 \% \text {, } \\
\downarrow \text { small fiber in } 23 \% \\
\text { 2. } \downarrow \text { Sural SNAP in } 27 \% \\
\text { 3. Sensory symptoms or } \\
\text { signs in } 32 \%\end{array}$ \\
\hline Weis J. (64) & $28 / 17$ & Skin biopsy & $\downarrow$ IENFD in $79 \%$ vs $12 \%$ \\
\hline Dalla Bella E. (66) & 57 & Skin biopsy & $\begin{array}{l}\downarrow \text { IENFD in } 75.4 \% \text { of } \\
\text { ALS and } 50 \% \text { of } \\
\text { FOSMN patients }\end{array}$ \\
\hline Ferrari G. (68) & $8 / 7$ & CCM & $\downarrow$ Mean CNFD \\
\hline
\end{tabular}


MCD: Meissner's corpuscle density, SGNFD: sweat gland nerve fiber density, PNFD: pilomotor nerve fiber density, FOSMN: Facial onset sensory and motor neuronopathy

\section{Abbreviations:}

ALS: amyotrophic lateral sclerosis; CIDP: chronic inflammatory demyelinating polyneuropathy; CCM: corneal confocal microscopy; EDS: Ehlers-Danlos syndrome; FM: fibromyalgia; GBS: Guillain-Barré syndrome; HPV: human papillomavirus; IENFD: intraepidermal nerve fiber density; IGT: impaired glucose tolerance; PD: Parkinson Disease; RSD: REM sleep behavior disorder; SFN: small fiber neuropathy

\section{References}

1. Hoeijmakers JG, Faber CG, Lauria G, Merkies IS, Waxman SG. Small-fibre neuropathies-advances in diagnosis, pathophysiology and management. Nat Rev Neurol. 2012;8(7):369-79.

2. Wooten M, Weng HJ, Hartke TV, Borzan J, Klein AH, Turnquist B, et al. Three functionally distinct classes of C-fibre nociceptors in primates. Nature Commun. 2014;5:4122.

3. Gasser HS. The classification of nerve fibers. 1941.

4. Peters MJ, Bakkers M, Merkies IS, Hoeijmakers JG, van Raak EP, Faber CG. Incidence and prevalence of small-fiber neuropathy: a survey in the Netherlands. Neurology. 2013;81(15):1356-60.

5. Burke JF, Skolarus LE, Callaghan BC, Kerber KA. Choosing Wisely: highest-cost tests in outpatient neurology. Ann Neurol. 2013;73(5):679-83.

6. Bouhassira D, Lantéri-Minet M, Attal N, Laurent B, Touboul C. Prevalence of chronic pain with neuropathic characteristics in the general population. Pain. 2008;136(3):380-7.

7. Uceyler N, Kafke W, Riediger N, He L, Necula G, Toyka KV, et al. Elevated proinflammatory cytokine expression in affected skin in small fiber neuropathy. Neurology. 2010;74(22):1806-13.

8. Cunha FQ, Poole S, Lorenzetti BB, Ferreira SH. The pivotal role of tumour necrosis factor alpha in the development of inflammatory hyperalgesia. Br J Pharmacol 1992;107(3):660-4.

9. Russell FA, Fernandes ES, Courade JP, Keeble JE, Brain SD. Tumour necrosis factor alpha mediates transient receptor potential vanilloid 1-dependent bilateral thermal hyperalgesia with distinct peripheral roles of interleukin-1beta, protein kinase $C$ and cyclooxygenase- 2 signalling. Pain. 2009;142(3):264-74.

10. Bednarik J, Vlckova-Moravcova E, Bursova S, Belobradkova J, Dusek L, Sommer C. Etiology of small-fiber neuropathy. J Peripher Nerv Syst. 2009;14(3):177-83.

11. Devigili G, Tugnoli V, Penza P, Camozzi F, Lombardi R, Melli G, et al. The diagnostic criteria for small fibre neuropathy: from symptoms to neuropathology. Brain 2008;131(Pt 7):1912-25.

12. Emery EC, Luiz AP, Wood JN. Nav1.7 and other voltage-gated sodium channels as drug targets for pain relief. Expert opinion on therapeutic targets. 2016;20(8):975-83. 
13. de Greef BTA, Hoeijmakers JGJ, Gorissen-Brouwers CML, Geerts M, Faber CG, Merkies ISJ. Associated conditions in small fiber neuropathy - a large cohort study and review of the literature. Eur J Neurol. 2018;25(2):348-55.

14. P T. Quantitative Sensory Testing. In: (Eds) Kea, editor. Neuromuscular Disorders in Clinical Practice. 1. New York: Springer Science + Business Media; 2014. p. 223-33.

15. Lauria G, Hsieh ST, Johansson O, Kennedy WR, Leger JM, Mellgren SI, et al. European Federation of Neurological Societies/Peripheral Nerve Society Guideline on the use of skin biopsy in the diagnosis of small fiber neuropathy. Report of a joint task force of the European Federation of Neurological Societies and the Peripheral Nerve Society. Eur J Neurol 2010;17(7):903-12, e44-9.

16. Lauria G, Bakkers M, Schmitz C, Lombardi R, Penza P, Devigili G, et al. Intraepidermal nerve fiber density at the distal leg: a worldwide normative reference study. J Peripher Nerv Syst 2010;15(3):202-7.

17. Petropoulos IN, Ponirakis G, Khan A, Gad H, Almuhannadi H, Brines M, et al. Corneal confocal microscopy: ready for prime time. Clin Exp Optom 2019.

18. Gemignani F. Small fiber neuropathy or small fiber pathology? Pain. 2017;158(5):988-9. 19. Uceyler N. Small fiber pathology--a culprit for many painful disorders? Pain. 2016;157 Suppl 1:S60-6.

20. Gibbons $\mathrm{CH}$, Schmidt P, Biaggioni I, Frazier-Mills C, Freeman R, Isaacson S, et al. The recommendations of a consensus panel for the screening, diagnosis, and treatment of neurogenic orthostatic hypotension and associated supine hypertension. J Neurol 2017.

21. Wolfe F, Clauw DJ, Fitzcharles MA, Goldenberg DL, Katz RS, Mease P, et al. The American College of Rheumatology preliminary diagnostic criteria for fibromyalgia and measurement of symptom severity. Arthritis Care Res (Hoboken) 2010;62(5):600-10.

22. Sarzi-Puttini P, Atzeni FJA. Fibromyalgia: A Never-Ending Story of Central and Peripheral Pain Mechanisms. Arthritis Rheumatol 2014;66(7):1687-8.

23. Jensen KB, Loitoile R, Kosek E, Petzke F, Carville $S$, Fransson P, et al. Patients with fibromyalgia display less functional connectivity in the brain's pain inhibitory network. Mol Pain. 2012;8:32.

24. Kim S-HJMh. Skin biopsy findings: implications for the pathophysiology of fibromyalgia. 2007;69(1):141-4.

25. Giannoccaro MP, Donadio V, Incensi A, Avoni P, Liguori R. Small nerve fiber involvement in patients referred for fibromyalgia. Muscle Nerve. 2014;49(5):757-9.

26. Caro XJ, Winter EF, Dumas AJ. A subset of fibromyalgia patients have findings suggestive of chronic inflammatory demyelinating polyneuropathy and appear to respond to IVIg. Rheumatology (Oxford). 2008;47(2):208-11.

27. Uceyler N, Zeller D, Kahn AK, Kewenig S, Kittel-Schneider S, Schmid A, et al. Small fibre pathology in patients with fibromyalgia syndrome. Brain 2013;136(Pt 6):1857-67.

28. Kosmidis ML, Koutsogeorgopoulou L, Alexopoulos H, Mamali I, Vlachoyiannopoulos PG, Voulgarelis M, et al. Reduction of Intraepidermal Nerve Fiber Density (IENFD) in the skin biopsies of patients with fibromyalgia: a controlled study. J Neurol Sci 2014;347(1-2):143-7.

29. Caro XJ, Winter EF. Evidence of abnormal epidermal nerve fiber density in fibromyalgia: clinical and immunologic implications. Arthritis \& rheumatology (Hoboken). 2014;66(7):1945-54. 30. Doppler K, Rittner HL, Deckart M, Sommer C. Reduced dermal nerve fiber diameter in skin biopsies of patients with fibromyalgia. Pain. 2015;156(11):2319-25. 
31. Lawson VH, Grewal J, Hackshaw KV, Mongiovi PC, Stino AM. Fibromyalgia syndrome and small fiber, early or mild sensory polyneuropathy. Muscle Nerve. 2018;58(5):625-30.

32. Lodahl M, Treister R, Oaklander AL. Specific symptoms may discriminate between fibromyalgia patients with vs without objective test evidence of small-fiber polyneuropathy. Pain reports. 2018;3(1):e633.

33. Oudejans L, He X, Niesters M, Dahan A, Brines M, van Velzen M. Cornea nerve fiber quantification and construction of phenotypes in patients with fibromyalgia. Sci Rep 2016;6:23573.

34. Ramirez M, Martinez-Martinez LA, Hernandez-Quintela E, Velazco-Casapia J, Vargas A, Martinez-Lavin M. Small fiber neuropathy in women with fibromyalgia. An in vivo assessment using corneal confocal bio-microscopy. Semin Arthritis Rheum 2015;45(2):214-9.

35. Erkan Turan K, Kocabeyoglu S, Unal-Cevik I, Bezci F, Akinci A, Irkec M. Ocular Surface Alterations in the Context of Corneal In Vivo Confocal Microscopic Characteristics in Patients With Fibromyalgia. Cornea. 2018;37(2):205-10.

36. Leinders M, Doppler K, Klein T, Deckart M, Rittner H, Sommer C, et al. Increased cutaneous miR-let-7d expression correlates with small nerve fiber pathology in patients with fibromyalgia syndrome. Pain. 2016;157(11):2493-503.

37. Evdokimov D, Frank J, Klitsch A, Unterecker S, Warrings B, Serra J, et al. Reduction of skin innervation is associated with a severe fibromyalgia phenotype. Ann Neurol. 2019;86(4):504-16.

38. Grayston R, Czanner G, Elhadd K, Goebel A, Frank B, Uceyler N, et al. A systematic review and meta-analysis of the prevalence of small fiber pathology in fibromyalgia: Implications for a new paradigm in fibromyalgia etiopathogenesis. Semin Arthritis Rheum 2019;48(5):933-40.

39. Sluka KA, Clauw DJJN. Neurobiology of fibromyalgia and chronic widespread pain. Neuroscience 2016;338:114-29.

40. Oaklander AL, Herzog ZD, Downs HM, Klein MM. Objective evidence that small-fiber polyneuropathy underlies some illnesses currently labeled as fibromyalgia. Pain. 2013;154(11):23106.

41. UKPDS. Intensive blood-glucose control with sulphonylureas or insulin compared with conventional treatment and risk of complications in patients with type 2 diabetes (UKPDS 33). UK Prospective Diabetes Study (UKPDS) Group. Lancet. 1998;352(9131):837-53.

42. Chaudhuri KR, Healy DG, Schapira AHJTLN. Non-motor symptoms of Parkinson's disease: diagnosis and management. Lancet Neurol 2006;5(3):235-45.

43. Rajabally YA, Martey JJN. Neuropathy in Parkinson disease: prevalence and determinants. Neurology 2011;77(22):1947-50.

44. Lökk JJL. Treatment with levodopa can affect latent vitamin B 12 and folic acid deficiency. Patients with Parkinson disease runt the risk of elevated homocysteine levels. 2003;100(35):2674-7. 45. Calzetti S, Bellanova MF, Negrotti A, Saccani E, Capozzi A, Pietrini V. Non-length-dependent somatosensory small fiber pathology presenting with restless legs syndrome in pre-motor Parkinson's disease. Evidence from skin biopsy in four patients. J Clin Neurosci 2019;69:139-42.

46. Adewusi JK, Hadjivassiliou M, Vinagre-Aragón A, O'Connor KR, Khan A, Grünewald RA, et al. Peripheral neuropathic pain in idiopathic Parkinson's disease: Prevalence and impact on quality of life; a case controlled study. J Neurol Sci 2018;392:3-7.

47. Nolano M, Provitera V, Estraneo A, Selim MM, Caporaso G, Stancanelli A, et al. Sensory deficit in Parkinson's disease: evidence of a cutaneous denervation. Brain 2008;131(Pt 7):1903-11. 
48. Lin CH, Chao CC, Wu SW, Hsieh PC, Feng FP, Lin YH, et al. Pathophysiology of Small-Fiber Sensory System in Parkinson's Disease: Skin Innervation and Contact Heat Evoked Potential. Medicine. 2016;95(10):e3058.

49. Podgorny PJ, Suchowersky O, Romanchuk KG, Feasby TE. Evidence for small fiber neuropathy in early Parkinson's disease. Parkinsonism Relat Disord 2016;28:94-9.

50. Kass-Iliyya L, Javed S, Gosal D, Kobylecki C, Marshall A, Petropoulos IN, et al. Small fiber neuropathy in Parkinson's disease: A clinical, pathological and corneal confocal microscopy study. Parkinsonism Relat Disord 2015;21(12):1454-60.

51. Nolano M, Provitera V, Manganelli F, lodice R, Stancanelli A, Caporaso G, et al. Loss of cutaneous large and small fibers in naive and I-dopa-treated PD patients. Neurology. 2017;89(8):77684.

52. Wang N, Gibbons $\mathrm{CH}$, Lafo J, Freeman R. alpha-Synuclein in cutaneous autonomic nerves. Neurology. 2013;81(18):1604-10.

53. Dauvilliers Y, Schenck CH, Postuma RB, Iranzo A, Luppi PH, Plazzi G, et al. REM sleep behaviour disorder. Nat Rev Dis Primers. 2018;4(1):19.

54. Donadio V, Doppler K, Incensi A, Kuzkina A, Janzen A, Mayer G, et al. Abnormal alphasynuclein deposits in skin nerves: intra- and inter-laboratory reproducibility. Eur J Neurol 2019.

55. Strobel AV, Tankisi H, Finnerup NB, Fuglsang-Frederiksen A, Jennum P, Svendsen KB, et al. Somatosensory function is impaired in patients with idiopathic REM sleep behaviour disorder. Sleep Med 2018;42:83-9.

56. Schrempf W, Katona I, Dogan I, Felbert VV, Wienecke M, Heller J, et al. Reduced intraepidermal nerve fiber density in patients with REM sleep behavior disorder. Parkinsonism Relat Disord 2016;29:10-6.

57. Hammad M, Silva A, Glass J, Sladky J, Benatar MJN. Clinical, electrophysiologic, and pathologic evidence for sensory abnormalities in ALS. Neurology 2007;69(24):2236-42.

58. Ishida N, Hongo S, Kumano A, Hatta H, Zakoji N, Hirutani M, et al. Relationship between Pain and Functional Status in Patients with Amyotrophic Lateral Sclerosis: A Multicenter Cross-Sectional Study. J Palliat Med 2018;21(5):588-91.

59. Chio A, Canosa A, Gallo S, Moglia C, llardi A, Cammarosano S, et al. Pain in amyotrophic lateral sclerosis: a population-based controlled study. Eur J Neurol 2012;19(4):551-5.

60. Chio A, Mora G, Lauria G. Pain in amyotrophic lateral sclerosis. Lancet Neurol. 2017;16(2):144-57.

61. Kerkhoff $\mathrm{H}$, Jennekens FG, Troost $\mathrm{D}$, Veldman $\mathrm{H}$. Nerve growth factor receptor immunostaining in the spinal cord and peripheral nerves in amyotrophic lateral sclerosis. Acta Neuropathol. 1991;81(6):649-56.

62. Luigetti M, Conte A, Del Grande A, Bisogni G, Romano A, Sabatelli M. Sural nerve pathology in ALS patients: a single-centre experience. Neurol Sci. 2012;33(5):1095-9.

63. Hammad M, Silva A, Glass J, Sladky JT, Benatar M. Clinical, electrophysiologic, and pathologic evidence for sensory abnormalities in ALS. Neurology. 2007;69(24):2236-42.

64. Weis J, Katona I, Muller-Newen G, Sommer C, Necula G, Hendrich C, et al. Small-fiber neuropathy in patients with ALS. Neurology. 2011;76(23):2024-9.

65. Truini A, Biasiotta A, Onesti E, Di Stefano G, Ceccanti M, La Cesa S, et al. Small-fibre neuropathy related to bulbar and spinal-onset in patients with ALS. J Neurol 2015;262(4):1014-8.

66. Dalla Bella E, Lombardi R, Porretta-Serapiglia C, Ciano C, Gellera C, Pensato V, et al. Amyotrophic lateral sclerosis causes small fiber pathology. Eur J Neurol 2016;23(2):416-20. 
67. Ren Y, Liu W, Li Y, Sun B, Li Y, Yang F, et al. Cutaneous somatic and autonomic nerve TDP-43 deposition in amyotrophic lateral sclerosis. J Neurol 2018;265(8):1753-63.

68. Ferrari G, Grisan E, Scarpa F, Fazio R, Comola M, Quattrini A, et al. Corneal confocal microscopy reveals trigeminal small sensory fiber neuropathy in amyotrophic lateral sclerosis. Front Aging Neurosci 2014;6:278.

69. Beighton P, De Paepe A, Steinmann B, Tsipouras P, Wenstrup RJ. Ehlers-Danlos syndromes: revised nosology, Villefranche, 1997. Ehlers-Danlos National Foundation (USA) and Ehlers-Danlos Support Group (UK). Am J Med Genet 1998;77(1):31-7.

70. Camerota F, Celletti C, Castori M, Grammatico P, Padua L. Neuropathic pain is a common feature in Ehlers-Danlos syndrome. J Pain Symptom Manag 2011;41(1):e2-4.

71. Rombaut L, Scheper M, De Wandele I, De Vries J, Meeus M, Malfait F, et al. Chronic pain in patients with the hypermobility type of Ehlers-Danlos syndrome: evidence for generalized hyperalgesia. Clini Rheumatol 2015;34(6):1121-9.

72. Cazzato D, Castori M, Lombardi R, Caravello F, Bella ED, Petrucci A, et al. Small fiber neuropathy is a common feature of Ehlers-Danlos syndromes. Neurology. 2016;87(2):155-9.

73. Rodriguez-Galan MA, Perez-Vilar S, Diez-Domingo J, Tuells J, Gomar-Fayos J, Morales-Olivas $\mathrm{F}$, et al. [Adverse reactions to human papillomavirus vaccine in the Valencian Community (20072011)]. An Pediatr (Barc). 2014;81(5):303-9.

74. Blitshteyn S. Postural tachycardia syndrome following human papillomavirus vaccination. Eur J Neurol 2014;21(1):135-9.

75. Souayah N, Ajroud-Driss S, Sander HW, Brannagan TH, Hays AP, Chin RL. Small fiber neuropathy following vaccination for rabies, varicella or Lyme disease. Vaccine. 2009;27(52):7322-5.

76. Gibbels E, Kentenich MJAn. Unmyelinated fibers in sural nerve biopsies of chronic inflammatory demyelinating polyneuropathy. Acta Neuropathol 1990;80(4):439-47.

77. Schneider C, Bucher F, Cursiefen C, Fink GR, Heindl LM, Lehmann HCJJotPNS. Corneal confocal microscopy detects small fiber damage in chronic inflammatory demyelinating polyneuropathy (CIDP). J Peripher Nerv Syst 2014;19(4):322-7.

78. Martinez V, Fletcher D, Martin F, Orlikowski D, Sharshar T, Chauvin M, et al. Small fibre impairment predicts neuropathic pain in Guillain-Barre syndrome. Pain. 2010;151(1):53-60.

79. Thomaides TN, Kerezoudi EP, Zoukos Y, Chaudhuri KR. Thermal thresholds and motor sensory conduction measurements in Guillain Barre syndrome: 12-month follow-up study. Eur Neurol 1992;32(5):274-80.

80. Pan CL, Tseng TJ, Lin YH, Chiang MC, Lin WM, Hsieh ST. Cutaneous innervation in GuillainBarre syndrome: pathology and clinical correlations. Brain 2003;126(Pt 2):386-97.

81. Seneviratne $U$, Gunasekera $S$. Acute small fibre sensory neuropathy: another variant of Guillain-Barre syndrome? J Neurol Neurosurg Psychiatry 2002;72(4):540-2.

82. Chiang MC, Lin YH, Pan CL, Tseng TJ, Lin WM, Hsieh ST. Cutaneous innervation in chronic inflammatory demyelinating polyneuropathy. Neurology. 2002;59(7):1094-8.

83. Figueroa JJ, Dyck PJ, Laughlin RS, Mercado JA, Massie R, Sandroni P, et al. Autonomic dysfunction in chronic inflammatory demyelinating polyradiculoneuropathy. Neurology. 2012;78(10):702-8.

84. Luigetti M, Madia F, Conte A, Tonali P, Sabatelli M. Neuropathy with predominant small fiber involvement associated with abnormal anti-MAG titer. Internal Med. 2010;49(23):2627-9. 
85. Rajabally YA, Delmont E, Hiew FL, Aube-Nathier AC, Grapperon AM, Cassereau J, et al. Prevalence, correlates and impact of pain and cramps in anti-MAG neuropathy: a multicentre European study. Eur J Neurol 2018;25(1):135-41.

86. Emery EC, Young GT, Berrocoso EM, Chen L, McNaughton PA. HCN2 ion channels play a central role in inflammatory and neuropathic pain. Science (New York, NY). 2011;333(6048):1462-6. 87. Han C, Hoeijmakers JG, Ahn HS, Zhao P, Shah P, Lauria G, et al. Nav1.7-related small fiber neuropathy: impaired slow-inactivation and DRG neuron hyperexcitability. Neurology. 2012;78(21):1635-43.

88. Klein CJ, Lennon VA, Aston PA, McKeon A, Pittock SJ. Chronic pain as a manifestation of potassium channel-complex autoimmunity. Neurology. 2012;79(11):1136-44.

89. Favoni V, Liguori R, Incensi A, Fileccia E, Donadio V. The incidental finding of elevated anti GQ1B antibodies in a patient with selective small fiber neuropathy. J Neurol Sci 2018;388:192-4.

90. Giannoccaro MP, Donadio V, Incensi A, Pizza F, Cason E, Di Stasi V, et al. Skin biopsy and I123 MIBG scintigraphy findings in idiopathic Parkinson's disease and parkinsonism: a comparative study. Mov Disord 2015;30(7):986-9.

91. Dabby R, Djaldetti R, Shahmurov M, Treves TA, Gabai B, Melamed E, et al. Skin biopsy for assessment of autonomic denervation in Parkinson's disease. J Neural Transm 2006;113(9):1169-76.

92. Nolano M, Provitera V, Stancanelli A, Saltalamacchia AM, Caporaso G, Lullo F, et al. Small fiber pathology parallels disease progression in Parkinson disease: a longitudinal study. Acta Neuropathol 2018;136(3):501-503.

93. Sassone J, Taiana M, Lombardi R, Porretta-Serapiglia C, Freschi M, Bonanno S, et al. ALS mouse model SOD1G93A displays early pathology of sensory small fibers associated to accumulation of a neurotoxic splice variant of peripherin. Hum Mol Genet 2016;25(8):1588-99. 